\title{
Azathioprine-induced pericarditis in a patient with ulcerative colitis
}

\author{
C DAVID SIMPSON MD
}

CD SIMPSON. Azathioprine-induced pericarditis in a patient with ulcerative colitis. Can J Gastroenterol 1997;11(3):217219. Inflammatory bowel disease is rarely associated with pericarditis but both sulfasalazine and the aminosalicylates have been known to cause this complication. To the author's knowledge this is the first reported case of acute pericarditis caused by azathioprine. It is believed that pericarditis is yet another potential manifestation of hypersensitivity to this drug.

Key Words: Azathioprine, Hypersensitivity, Pericarditis, Ulcerative colitis

A zathioprine, a purine analogue, has been used for many years in the treatment of idiopathic inflammatory bowel disease (IBD). Despite the development of newer immunomodulatory agents that are useful in acutely ill patients, azathioprine retains a place in the management of patients requiring a persistently high dose of corticosteroids for disease control (1). Although a number of potentially serious adverse effects of the purine analogues are well known, studies assessing toxicity of azathioprine in rheumatoid arthritis (2) and 6-mercaptopurine (the active metabolite of azathioprine) in IBD (3) have shown a surprisingly low incidence of serious toxicity. To our knowledge this is the first reported case of azathioprine-induced pericarditis in a patient with ulcerative colitis.

\section{Péricardite induite par l'azathioprine chez un patient atteint de colite ulcéreuse}

RÉSUMÉ : La maladie inflammatoire de l'intestin est rarement associée à une péricardite mais on sait que la sulfasalazine et les aminosalicylates peuvent causer cette complication. À la connaissance de l'auteur, le cas décrit dans cet article est le premier cas rapporté de péricardite aiguë attribuable à l'azathioprine. On croit que la péricardite est encore un autre signe potentiel d'hypersensibilité à ce médicament. 


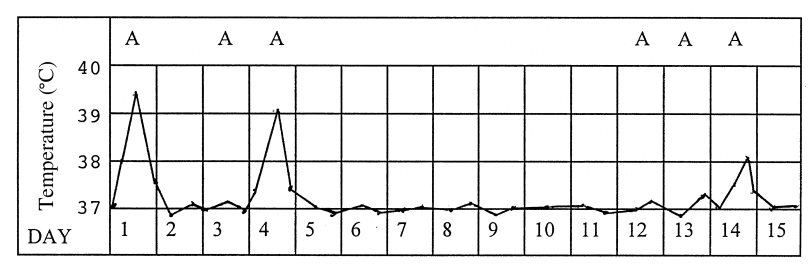

Figure 1) Temperature curve starting on the day a pericardial rub was first noted. 'A' indicates azathioprine was given that day

he was started on azathioprine $100 \mathrm{mg} /$ day two weeks before presentation. Other medications were prednisone $20 \mathrm{mg} /$ day, mesalamine $1200 \mathrm{mg}$ tid and ranitidine $150 \mathrm{mg}$ bid.

Ten days after starting the azathioprine he felt vaguely unwell with low back pain and fatigue. Over the ensuing three days he developed worsening back pain, headache, diaphoresis and chills. The night before admission he developed severe arthralgias involving the knees, hips and ankles without any frank signs of arthritis. His colitic symptoms were unchanged with eight to 10 daily episodes of small volume diarrhea with hematochezia. On examination at admission he had a temperature of $38^{\circ} \mathrm{C}$ and appeared cushingoid. Respiratory, cardiovascular, abdominal and musculoskeletal examinations were all normal. Routine laboratory investigations were normal except for a mild increase in liver enzymes: gamma-glutamyl transpeptidase $159 \mathrm{U} / \mathrm{L}$ (normal 10 to 50), alkaline phosphatase $158 \mathrm{U} / \mathrm{L}$ (normal 40 to 105 ), aspartate aminotransferase $96 \mathrm{U} / \mathrm{L}$ (normal 10 to 35 ) and alanine aminotransferase $86 \mathrm{U} / \mathrm{L}$ (normal 10 to 35). Bilirubin was normal. Chest $\mathrm{x}$-ray was normal and cultures of throat, urine, stool and blood were negative. Viral serology was not obtained.

The day after admission the patient developed anterior pleuritic chest pain for the first time and a pericardial friction rub was heard. Serial electrocardiograms showed only nonspecific ST-T changes. Creatine phosphokinase values were normal. An echocardiogram done five days after the onset of the rub showed a small pericardial effusion, confirming the clinical diagnosis of acute pericarditis. The patient's azathioprine medication was held for one day; on that day he felt better with no further chest pain and a marked reduction in arthralgias. When a review of standard reference texts did not reveal any known association between pericarditis and azathioprine, azathioprine was restarted. Two days later he again developed fever, pleuritic chest pain and arthralgias, and azathioprine was discontinued. Over the ensuing $24 \mathrm{~h}$ his pleuritic chest pain, pericardial rub, arthralgias and fever all resolved and he felt well over the next week aside from his ongoing colitic symptoms. During this whole period the mesalamine and prednisone were continued. Rheumatoid factor was negative and antinuclear antibody level was not significant with a titre of $1 / 100$. A literature search produced no reports of an association between azathioprine and pericarditis, and therefore the possibility of coincidental idiopathic or viral pericarditis was considered. The literature search was conducted using the MEDLINE database (1982 to October 1991) using the key words 'azathioprine', 'hypersensitivity' and 'pericarditis'. A manual search of the reference lists from relevant articles was also conducted.

Because the patient did not want surgery, azathioprine therapy was restarted after he had been asymptomatic while not taking that drug for one week. Over the next two days he developed a low grade fever, and on the third day he again developed pleuritic chest pain and a pericardial rub. Azathioprine was stopped and these symptoms and signs resolved over $24 \mathrm{~h}$ and did not recur. Figure 1 shows his temperature curve in relation to azathioprine dosing. Subsequently it continued to prove impossible to wean the patient from systemic steroids, and he ultimately underwent colectomy with good results.

\section{DISCUSSION}

Although there have been rare case reports of pericarditis as an extra-intestinal manifestation of IBD unrelated to medication (4), the clinical course in this case provides conclusive evidence that azathioprine was the cause of pericarditis. A number of drugs are well documented to cause pericarditis. There is a relatively high incidence of hypersensitivity reactions, including pericarditis, to sulfasalazine (5) but there have also been a number of reports of pericarditis caused by mesalamine either because of a hypersensitivity reaction (6) or because of the development of a lupus-like syndrome (7). Although the aminosalicylates have far fewer adverse effects than sulfasalazine, the drug they were originally designed to replace, it is becoming increasingly clear that they can provoke a number of adverse clinical syndromes, including acute pancreatitis (8). However, the clinical course in this case makes it impossible to implicate mesalamine as the cause of the pericarditis.

Azathioprine is well known to cause a number of infrequent but significant adverse effects, such as bone marrow suppression. As well, azathioprine hypersensitivity is well recognized although uncommon. Hypersensitivity is seen most often in patients with immune-mediated diseases and is usually associated with fever; symptoms typically resolve readily once the drug is discontinued. The onset of symptoms usually occurs between one and two weeks after starting the drug, but on rechallenge symptoms may develop rapidly and be more severe. The clinical picture can be quite variable although chills, arthralgias and myalgias commonly accompany fever. The clinical course in this case is typical for a hypersensitivity reaction to azathioprine (9), and this is believed to be the mechanism for the development of pericarditis. There have been case reports of a wide variety of clinical syndromes associated with the common manifestations of azathioprine hypersensitivity, including a number of gastrointestinal syndromes such as pancreatitis (9) and cholestatic jaundice (10).

Azathioprine hypersensitivity mimicking potential complications of underlying IBD has been reported (11). A recent meta-analysis of azathioprine and 6-mercaptopurine in Crohn's disease found that, when specified, the most com- 
mon adverse effects were allergic reactions consisting of fever and/or rash and arthritis in $2 \%$ of patients (12).

The cause and mechanism of azathioprine hypersensitivity are unknown. Immune mechanisms have been postulated, and the clinical features of most reported cases are consistent with an allergic etiology (13). The size of the azathioprine molecule makes it a poor immunogen, raising the possibility that it acts as a hapten. It is intriguing that there are case reports of such a wide variety of clinical syndromes in association with what appear to be the more typical manifestations of azathioprine hypersensitivity. Also, reported cases have occurred despite the concomitant use of

\section{REFERENCES}

1. Shanahan F, O'Sullivan GC, Collins JK. Immunosuppressive agents in inflammatory bowel disease: Current status and future prospects. Can J Gastroenterol 1994;8:383-7.

2. Singh G, Fries JF, Spitz P, Williams CA. Toxic effects of azathioprine in rheumatoid arthritis A national post-marketing perspective. Arthritis Rheum 1989;32:837-43.

3. Present DH, Meltzer SJ, Krumholz MP, Wolke A, Korelitz BI. 6-Mercaptopurine in the management of inflammatory bowel dissease: short- and long-term toxicity. Ann Intern Med 1989;111:641-9.

4. Becker SA, Wishnitzer R, Botwin S, Eliraz A, Bass DD. Myopericarditis associated with inflammatory bowel disease. J Clin Gastroenterol 1981;3:267-70.

5. Podolsky DK. Inflammatory bowel disease. N Engl J Med 1991;325:1008-16.

6. Lim AG, Hine KR. Fever, vasculitic rash, arthritis, pericarditis, and pericardial effusion after mesalazine. BMJ 1994;308:113. (Lett) systemic steroids in doses as high as prednisone $45 \mathrm{mg} /$ day (9).

There have been many cases of azathioprine hypersensitivity associated with hypotension, possibly due to cytokine release (13). Specific associated cardiac syndromes are exceedingly rare. Having extended the literature search outlined in the 'Case Presentation' to include up to June 1996, I conclude that this is the first reported case of pericarditis caused by azathioprine. This case highlights the necessity of considering the diagnostic possibility of azathioprine hypersensitivity in any patient being treated with this drug who develops any febrile illness soon after institution of therapy.

7. Dent MT, Ganapathy S, Holdsworth CD, Channer KC. Mesalazine induced lupus-like syndrome. BMJ 1992;305:159. (Lett)

8. Sachedina B, Saibil F, Cohen LB, Whittey J. Acute pancreatitis due to 5-aminosalicylate. Ann Intern Med 1989;110:490-2.

9. Saway PA, Heck LW, Bonner JR, Kirklin JK. Azathioprine hypersensitivity - Case report and review of the literature. Am J Med 1988;84:960-4.

10. Jeurissen MEC, Boerbooms AM Th, van de Putte LBA, Krujsen MWM. Azathioprine induced fever, chills, rash, and hepatotoxicity in rheumatoid arthritis. Ann Rheum Dis 1990;49:25-7.

11. Wilson BE, Parsonnet J. Azathioprine hypersensitivity mimicking sepsis in a patient with Crohn's disease. Clin Infect Dis 1993;17:940-1. (Lett)

12. Pearson DC, May GR, Fick GH, Sutherland LR. Azathioprine and 6-mercaptopurine in Crohn's disease. A meta-analysis. Ann Intern Med 1995; $122: 132-42$.

13. Knowles SR, Gupta AK, Shear NH, Sauder D. Azathioprine hypersensitivity-like reactions - a case report and a review of the literature. Clin Exp Dermatol 1995;20:353-6. 


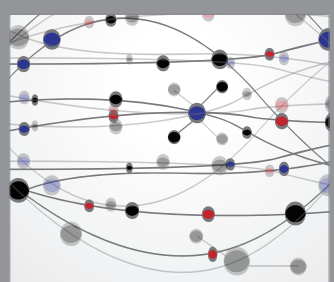

The Scientific World Journal
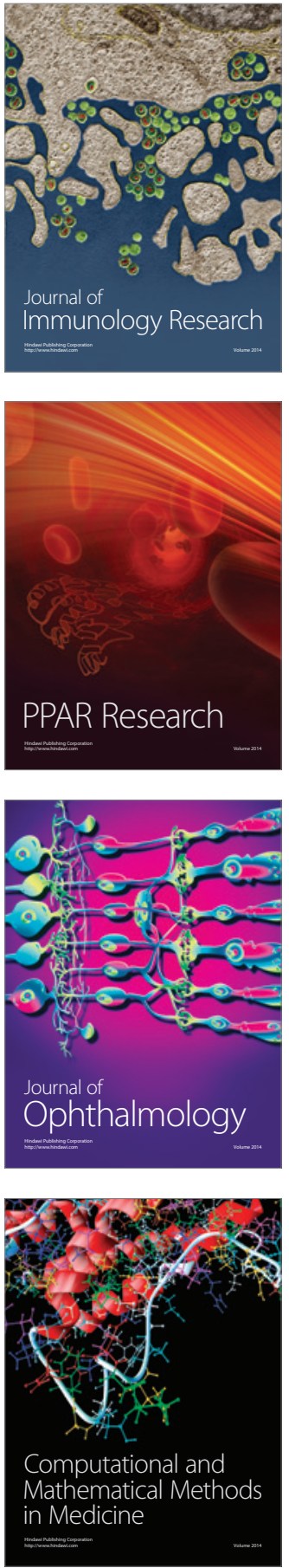

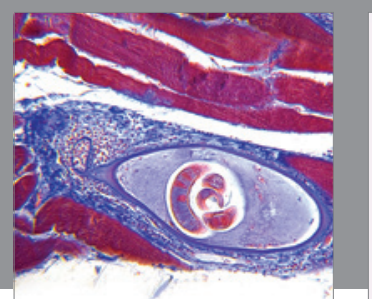

Gastroenterology Research and Practice

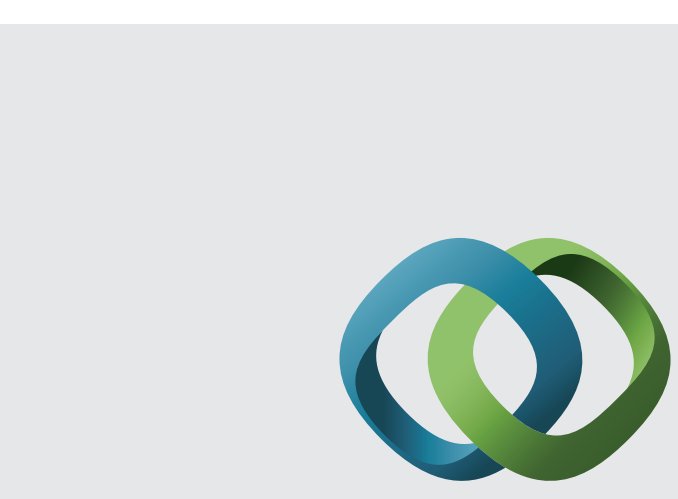

\section{Hindawi}

Submit your manuscripts at

http://www.hindawi.com
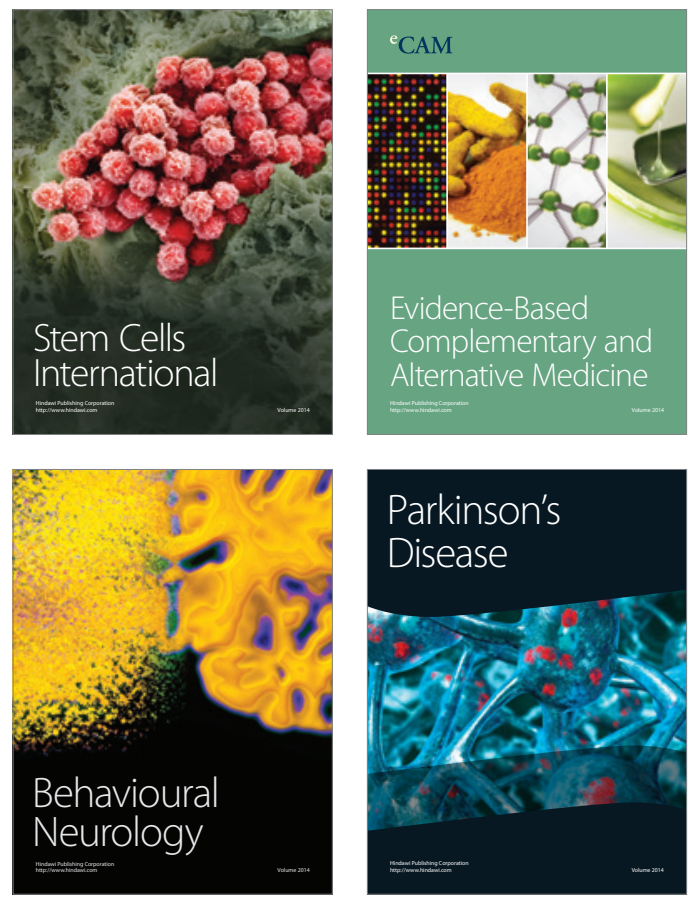
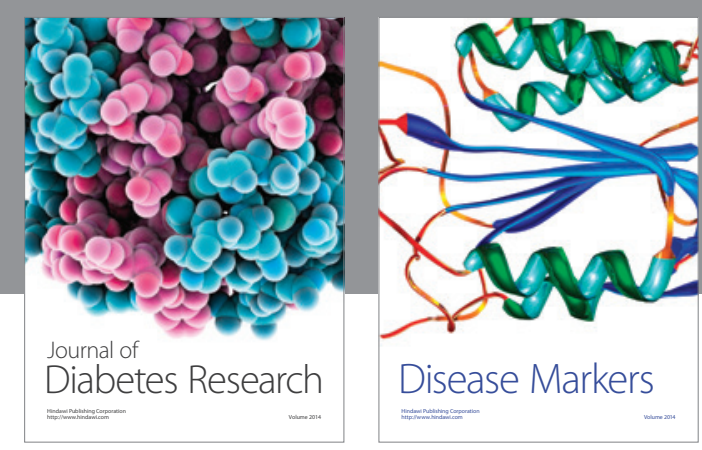

Disease Markers
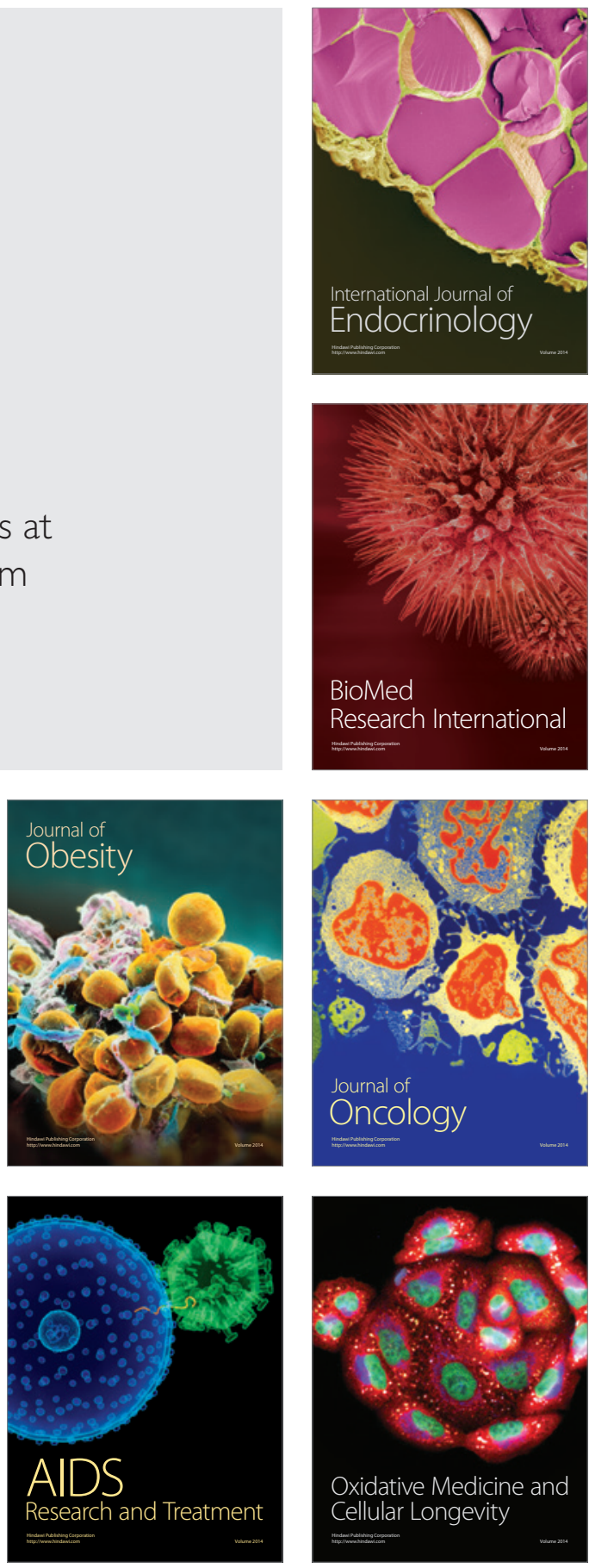